\title{
Individual Attention Patterns in Children Born Very Preterm and Full Term at 7 and 13 Years of Age
}

\author{
Lilly Bogičević ${ }^{1}$ (1) ,Leona Pascoe ${ }^{2,3}$, Thi-Nhu-Ngoc Nguyen ${ }^{2,3}$, Alice C. Burnett ${ }^{3,4,5,6}$, Marjolein Verhoeven ${ }^{1}$, \\ Deanne K. Thompson ${ }^{3,6,7,8}$, Jeanie L.Y. Cheong ${ }^{3,5,9}$, Terrie E. Inder ${ }^{10}$, Anneloes L. van Baar ${ }^{1}$, Lex W. Doyle ${ }^{3,5,6,9}$ and \\ Peter J. Anderson ${ }^{2,3}$ \\ ${ }^{1}$ Child and Adolescent Studies, Utrecht University, Utrecht, The Netherlands \\ ${ }^{2}$ Turner Institute for Brain and Mental Health, School of Psychological Sciences, Monash University, Melbourne, Victoria, Australia \\ ${ }^{3}$ Victorian Infant Brain Studies (VIBeS), Murdoch Children's Research Institute, Melbourne, Victoria, Australia \\ ${ }^{4}$ Royal Children's Hospital, Melbourne, Victoria, Australia \\ ${ }^{5}$ Neonatal Services, Royal Women's Hospital, Melbourne, Victoria, Australia \\ ${ }^{6}$ Department of Paediatrics, University of Melbourne, Melbourne, Victoria, Australia \\ ${ }^{7}$ Developmental Imaging, Murdoch Children's Research Institute, Melbourne, Victoria, Australia \\ ${ }^{8}$ Florey Institute of Neuroscience and Mental Health, Melbourne, Victoria, Australia \\ ${ }^{9}$ Department of Obstetrics and Gynaecology, University of Melbourne, Melbourne, Victoria, Australia \\ ${ }^{10}$ Brigham and Women's Hospital, Harvard Medical School, Boston, MA, USA
}

(Received July 6, 2020; Final revision November 11, 2020; AcCePted December 29, 2020; First Published Online January 22, 2021)

\begin{abstract}
Objective: To identify attention profiles at 7 and 13 years, and transitions in attention profiles over time in children born very preterm (VP; $<30$ weeks' gestation) and full term (FT), and examine predictors of attention profiles and transitions. Methods: Participants were 167 VP and 60 FT children, evaluated on profiles across five attention domains (selective, shifting and divided attention, processing speed, and behavioral attention) at 7 and 13 years using latent profile analyses. Transitions in profiles were assessed with contingency tables. For VP children, biological and social risk factors were tested as predictors with a multinomial logistic regression. Results: At 7 and 13 years, three distinct profiles of attentional functioning were identified. VP children were 2-3 times more likely to show poorer attention profiles compared with FT children. Transition patterns between 7 and 13 years were stable average, stable low, improving, and declining attention. VP children were two times less likely to have a stable average attention pattern and three times more likely to have stable low or improving attention patterns compared with FT children. Groups did not differ in declining attention patterns. For VP children, brain abnormalities on neonatal MRI and greater social risk at 7 years predicted stable low or changing attention patterns over time. Conclusions: VP children show greater variability in attention profiles and transition patterns than FT children, with almost half of the VP children showing adverse attention patterns over time. Early brain pathology and social environment are markers for attentional functioning.
\end{abstract}

Keywords: Prematurity, Neurodevelopment, Longitudinal, Latent profile analysis, Trajectories, Risk factors

\section{INTRODUCTION}

Attention is a multidimensional construct, comprising separate yet inter-dependent cognitive and behavioral components. Children born very preterm (VP; <32 weeks' gestation) are at increased risk of impaired attentional functioning, performing poorer than children born full term (FT; $\geq 37$ weeks'

Correspondence and reprint requests to: Lilly Bogičević, Child and Adolescent Studies, Faculty of Social and Behavioural Sciences, Utrecht University, Langeveldgebouw, Heidelberglaan 1, 3584 CS Utrecht, The Netherlands. E-mail: 1.bogicevic@uu.nl. gestation) across selective, sustained, shifting, and divided attention domains (Bayless \& Stevenson, 2007; Lean, Melzer, Bora, Watts, \& Woodward, 2017; Mulder, Pitchford, \& Marlow, 2011). Compared with FT peers, VP children also show slower speed of information processing (Anderson \& Doyle, 2003; Rose, Feldman, Jankowski, \& van Rossem, 2011) which is an elementary skill often required in the assessment of attentional functioning (Mulder et al., 2009). Consistent with these findings, parents and teachers of VP children report more behavioral problems associated with attention, including distractibility and inattentiveness (Aarnoudse-Moens, Weisglas-Kuperus, van Goudoever, \& Oosterlaan, 2009). 
VP children are a heterogeneous group reflected in distinct profiles of functioning (Burnett et al., 2019; Lean et al., 2020). However, most studies have explored attentional functioning in VP children at group level compared with FT children, and little is known about individual variability of attention profiles. One recent study of 6-year-old children born moderate-to-late preterm (32-36 weeks' gestation) reported several distinct profiles of attention, including subgroups with average attention functioning, generalized attention difficulties, specific cognitive attention difficulties, and specific behavioral attention difficulties (Bogičević, Verhoeven, \& van Baar, 2020). However, such distinct profiles of attention have yet to be explored in children born VP, who are at increased risk of attention difficulties and may as well exhibit more individual variability in attention profiles.

Longitudinal studies of attention in children born VP are scarce. One longitudinal study showed that significant attention problems were still present in the VP group at 26 years of age, although parent-rated behavioral attention problems declined and observer-rated sustained attention improved between school-age and adulthood (6-26 years) (Breeman, Jaekel, Baumann, Bartmann, \& Wolke, 2016). Krasner et al. (2018) reported three distinct longitudinal profiles of Attention-Deficit/Hyperactivity Disorder (ADHD) symptoms from 6 to 16 years in a preterm born cohort. Longitudinal profiles included no ADHD symptoms across childhood, ADHD symptoms that declined by adolescence, and persistent inattentive symptoms with declining hyperactive/impulsive symptoms. A meta-analysis of cross-sectional studies investigating age at assessment and attentional functioning suggested that attention difficulties in children born preterm become most prominent from preschool age and may improve throughout later childhood in some, but not all, children born preterm compared with peers born FT (Mulder et al., 2009). While children born preterm may demonstrate some catch-up in specific attention domains with age, no study to date has explored changes in attention profiles across multiple attention domains over time.

This study addresses current gaps in the literature regarding attention profiles in children born VP. The primary aim was to identify and compare distinct attention profiles at 7 and at 13 years in VP and FT children and to assess individual variability of transitions in attention profiles between VP and FT children from 7 to 13 years. It was expected that VP children would show divergent attention profiles and would more often be assigned to poorer attention profiles than FT children at both 7 and 13 years of age. We also expected that some children would show stable attention profiles, but others would experience changing patterns of performance such as improved functioning, declining functioning, or a shift in type of attention difficulties. In addition, we expected VP children to display more variability in transitions in attention profiles from middle childhood to early adolescence compared with FT children. Furthermore, given that neonatal (e.g., lower birth weight and brain abnormalities) and social risk factors (e.g., lower parental education) have been previously associated with poorer attentional functioning (Eryigit-
Madzwamuse \& Wolke, 2015; Lean et al., 2017; McGrath et al., 2005; Murray et al., 2014), we examined whether these factors predicted transitions in profiles between 7 and 13 years in VP children.

\section{METHODS}

\section{Participants and Procedure}

Participants were children from the Victorian Infant Brain Study (VIBeS) cohort, comprising 224 children born VP and 46 children born FT between July 2001 and December 2003 at the Royal Women's Hospital, Melbourne, and an additional 31 FT children recruited at 2 years of age from maternal child health clinics. Children with severe genetic or congenital abnormalities known to affect neurodevelopment were excluded from the study.

Measures of attention were administered at 7 and 13 years by trained assessors who were blinded to birth status. For VP children, age was corrected for prematurity, that is, the child's chronological age was reduced by the number of weeks the child was born preterm, to allow VP children the same maturational time and to minimize a bias in cognitive test scores. The current study was restricted to participants for whom any attention data were available at both timepoints, as we were interested in changes in attention profiles across time within individuals. Informed consent was obtained from parents at both timepoints and the study was approved by the Human Research Ethics Committee at the Royal Women's Hospital and Royal Children's Hospital.

\section{Measures}

Selective attention, shifting attention, divided attention, sustained attention, processing speed, and behavioral attention were measured at 7 and 13 years of age. However, due to low ceiling of the sustained attention measure at 13 years, this domain was not reported or included in analyses.

\section{Attention at 7 years}

At 7 years, attention was assessed with three subtests from the Test of Everyday Attention in Children (TEA-Ch; Manly et al., 2001; Manly, Roberston, Anderson, \& Nimmo-Smith, 1999). Selective attention was assessed with Sky Search, requiring children to search for and circle relevant targets while ignoring irrelevant targets under time pressure. The total number of correct targets identified was the variable of interest. Shifting attention was assessed with Creature Counting, which required children to count visual targets and shift between responses (counting forward and counting backward) depending on a visual cue (arrow up or down). Performance was judged according to the number of correct trials. Divided attention was evaluated with the Sky Search Dual Task. Children were asked to complete the Sky Search task (as described above), while simultaneously counting the number of tones on an audio recording. Performance was determined by the proportion of accurate targets and counts (Anderson et al., 2011; Murray et al., 2014). 
Processing speed was examined with the baseline trial (Trial 1) of the Shape School test (Espy, 1997). Children were presented with several different shapes varying in color and were instructed to name the colors of each shape in correct order as fast and accurately as possible. An efficiency score was calculated, taking into account both time and accuracy (number of correct answers minus errors, divided by total time taken for the trial).

Behavioral attention was assessed with the Hyperactivity/ Inattention scale of the parent-reported Strength and Difficulties Questionnaire (SDQ; Goodman, 1997). Parents rated children's behavioral attention on five items as "not true," "somewhat true," or "certainly true." The scale was reverse coded so that lower scores indicated more behavioral attention problems.

\section{Attention at 13 years}

At 13 years, two subtests of the TEA-Ch were administered. Selective attention was evaluated with Map Mission, in which children were instructed to find and circle as many targets as possible within $1 \mathrm{~min}$ while ignoring irrelevant targets. The total number of correct targets was scored. Divided attention was assessed with Score! Dual Task, requiring children to count the number of tones on an audio recording while listening to a news report and remembering the name of the animal mentioned simultaneously. The total number of correct counts and animals was used.

Shifting attention and processing speed were evaluated using the Contingency Naming Test (Anderson, Anderson, Northam, \& Taylor, 2000). Processing speed was assessed using Trial 1, in which children were asked to name the color of a shape as fast and accurately as possible. Shifting attention was assessed using Trial 3, where children were required to name the color of the shape if the internal and external shapes were identical, and to name the external shape if it was different from the internal shape. In both trials, an efficiency score was computed, taking into account both time and accuracy (number of correct answers minus a square root transformation of errors, divided by total time taken for the trial).

Behavioral attention was assessed using the parentreported Hyperactivity/Inattention scale of the SDQ at 13 years (Goodman, 1997), which was reverse coded so that lower scores indicated more behavioral attention problems.

In order to create uniform scaling for each measure, raw scores were transferred into $z$-scores based on the mean and standard deviation $(S D)$ of the FT group. For all measures, higher scores indicated better attention performance with $z$-scores interpreted as follows: $\leq-2.0$ : extremely low, -2.0 to -1.5 : very low, -1.5 to -1.0 : low average, -1.0 to 1.0 : average, 1.0 to 1.5: high average, 1.5 to 2.0 very high, and $\geq 2.0$ : extremely high.

\section{Neonatal and social risk factors}

Predictors of the profiles assessed were biological and social risk factors. Biological factors of interest included gestational age, birth weight $z$-score (relative to sex and gestational age), bronchopulmonary dysplasia (oxygen dependency at 36 weeks' postmenstrual age, i.e., gestational age plus chronological age), neonatal infection (proven sepsis and/or necrotizing enterocolitis), major brain injury based on cranial ultrasound during the neonatal period (grade III/IV intraventricular hemorrhage and/or cystic periventricular leukomalacia), and overall brain abnormality as assessed on brain MRI at term-equivalent age (composite measure of white matter, cortical and deep grey matter, and cerebellar abnormalities; scores ranged from 0 to 40 with higher scores indicating more severe brain abnormality; Kidokoro, Neil \& Inder, 2013). Social risk was assessed at 7 years, based on family structure, primary caregiver education level, the primary income earner's occupation, the primary income earner's employment status, language spoken at home, and maternal age at birth (Roberts et al., 2008). These components were each scored on a threepoint scale (0-2) and summed to generate an overall social risk index (0-12), with scores $\geq 2$ categorized as higher social risk.

\section{Statistical Analyses}

To address our primary aim, the first step was to identify profiles of attention by performing separate latent profile analyses (LPA) at 7 and 13 years using the five measures of attention domains in Mplus 8.2 (Muthén \& Muthén, 2012). Missing data were handled using full information maximum likelihood, and both VP and FT children were included in the LPA models. For both timepoints, a series of LPA models specified with 1-profile to 5-profiles were examined and the best model was selected based on interpretability and fit indices. Fit indices included Bayesian Information Criterion (BIC), Adjusted Bayesian Information Criterion (Adj-BIC), and Akaike Information Criterion, where lower values indicated better fit, and the VuongLo-Mendell-Rubin and adjusted Lo-Mendell-Rubin model comparisons tests, with significant $p$-values indicating improved fit over the model with one less profile. Lastly, model entropy was examined with values closer to 1 indicative of good classification of individuals in subgroups (Berlin et al., 2014). To check for potential effects of missing data, LPAs were repeated for each timepoint including children who had data only at one timepoint.

After model selection, the second step involved assigning children to their most likely profile based on posterior probability values. Profile membership was used in subsequent analyses, performed in SPSS Statistics 25.0. Attention profiles were interpreted based on performance on all five attention domains. Given that sex and birth status (VP vs. FT) have previously been related to attentional functioning (Mulder et al., 2009), the distribution of these factors was compared across the different attention profiles with chi-square tests and post-hoc z-tests. Effect sizes were assessed with $\phi$ $($ small $=.10$, moderate $=.30$, large $=.50)$. 
In the third step, individual transitions between attention profiles from 7 to 13 years were examined for VP and FT children with contingency tables. VP and FT children were compared on change between attention profiles from 7 to 13 years with a chi-square test, as this test is not sensitive to small sample sizes and can be used with cells with low or zero counts. Based on profile membership at each timepoint, children were categorized into transition groups, such as stable average, stable low, improving, and declining attention functioning. To examine whether VP birth was associated with individual transitions between attention profiles, birth status was entered in a univariable multinomial logistic regression model.

To address our secondary aim, analyzing VP children only, we examined whether neonatal factors and social risk chosen a priori were associated with transition groups by performing a multivariable multinomial logistic regression. The regression model was assessed with $R^{2}$, and neonatal and social risk factors were examined using Wald tests.

\section{RESULTS}

\section{Participant Characteristics}

Participants' retention rates at the 7 and 13 year follow-ups are shown in Figure 1. Seven VP children were unable to complete some attention tasks due to very low IQ $(<70)$ at 7 and/or 13 years and were excluded from the analyses. The final sample comprised 167 (75\%) VP and 60 (78\%) FT children from the original cohorts. Of the final sample, three children taking prescribed stimulant medication at the time of the 7 year assessment were asked to withhold their morning dose. At the 13 year assessment, there were four children taking stimulant medication, though there was no direction to withhold it for the assessment. Therefore, LPAs at 13 years were repeated excluding these children. No differences were found between participants and nonparticipants (VP: $n=57$ and FT: $n=17$ ) in terms of background characteristics.

Participants' background characteristics and group mean performance on the five attention domains are shown in Table 1. The VP group performed poorer than the FT group on all measures of attention at 7 and 13 years with mean group differences ranging from .29 to $.74 S D$.

\section{Attention Profiles and Transitions from 7 to 13 Years}

Model fit indices for 1-to-5-profile models at 7 and 13 years for the total sample $(n=227)$ are shown in Table 2. At 7 years, all fit indices supported the 3-profile model (marked in bold). Fit indices also supported the 3-profile model at 13 years (marked in bold). Repeating LPAs including children for whom data were available only at $7(n=268)$ or 13 years and $(n=244)$ showed similar results and also favored the 3-profile models (Supplementary Table S1).

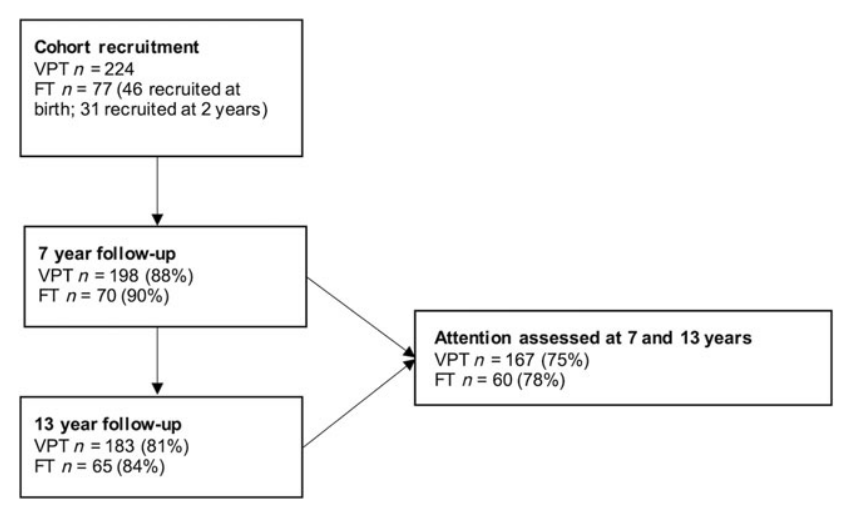

Fig. 1. Study flowchart.

Repeating LPAs excluding children taking stimulant medication at 13 years $(n=4)$ also favored the 3 -profile model at 13 years and did not alter interpretation of the profiles.

The three attention profiles at 7 years are illustrated in Figure 2A. Children in profile $7 \mathrm{a}$ showed average performance across all attention domains (z-scores: .01-.30). Profile $7 \mathrm{~b}$ was associated with very low performance on shifting attention $(z$-score: -1.60$)$ and low average to average performance on selective attention, divided attention, processing speed, and behavioral attention (z-scores: -1.04 to -.22 ). Profile $7 \mathrm{c}$ was characterized by extremely low performance on selective attention (z-score: -3.12 ) and low average to average performance across all other attention domains ( $z$-scores: -1.27 to -.95 ).

The three attention profiles at 13 years are illustrated in Figure 2B. Children in profile 13a showed average performance across all attention domains ( $z$-scores: -.56 to .35 ). Children assigned to profile $13 \mathrm{~b}$ had low average to average performance on all attention domains ( $z$-scores: -1.25 to -.55). Profile $13 \mathrm{c}$ was associated with extremely low performance on divided attention and processing speed ( $z$-scores: -3.16 and -2.23 ), very low shifting attention performance ( $z$-score: -1.78$)$, low average selective attention performance (z-score: -1.29$)$, and average behavioral attention functioning ( $z$-score: -.05$)$.

The distribution of sex and birth status (VP vs. FT) across the three profiles at 7 and 13 years is presented in Table 3. Sex was evenly distributed across the three profiles at 7 and 13 years of age. Most FT children were classified into profiles $7 \mathrm{a}(83 \%)$ and $13 \mathrm{a}(70 \%)$, representing the average attention functioning profiles. VP children were more dispersed across profiles and were overrepresented in the lower attention functioning profiles (i.e., $7 \mathrm{~b}, 7 \mathrm{c}, 13 \mathrm{~b}$, and 13c).

The transition of VP and FT individuals between attention profiles at 7 and 13 years is shown in Figure 3. Overall, change between attention profiles was more common in VP children compared with FT children, with a moderate effect size: $\chi^{2}(8,227)=29.49, p<.001, \phi=.36$. Concerning the average attention functioning profile at 7 years (7a), VP and FT children showed similar transitions, with the majority of children maintaining a profile of average attention functioning (13a). From the very low to average 
Table 1. Characteristics of VP and FT children

\begin{tabular}{|c|c|c|c|c|}
\hline & $\mathrm{VP}(n=167)$ & FT $(n=60)$ & Mean difference $(95 \% \mathrm{CI})$ & $p$-value \\
\hline \multicolumn{5}{|l|}{ Background characteristics } \\
\hline Male, $n(\%)$ & $86(52)$ & $27(45)$ & & \\
\hline Gestational age (weeks), M (SD) & $27.5(2.0)$ & $39.1(1.3)$ & & \\
\hline Birth weight $z$-score, $\mathrm{M}(S D)$ & $-.52(.91)$ & $.06(.95)$ & & \\
\hline Multiple birth (twin or triplet fetuses), $n(\%)$ & $77(46)$ & $4(7)$ & & \\
\hline Bronchopulmonary dysplasia, $n(\%)$ & $58(35)$ & 0 & & \\
\hline Neonatal infection, $n(\%)$ & $60(36)$ & 0 & & \\
\hline Cranial ultrasound major brain injury, $n(\%)$ & $12(7)$ & 0 & & \\
\hline MRI brain abnormalities, M $(S D)$ & $5.43(3.29)$ & $1.76(1.37)$ & & \\
\hline Higher social risk at 7 years, $n(\%)$ & $100(60)$ & $21(35)$ & & \\
\hline Age at 7 year follow-up, $\mathrm{M}(S D)$ & $7.51(.26)$ & $7.66(.32)$ & & \\
\hline Age at 13 year follow-up, $\mathrm{M}(S D)$ & $13.33(.61)$ & $13.12(.61)$ & & \\
\hline \multicolumn{5}{|l|}{ Attention domains (z-scores) } \\
\hline \multicolumn{5}{|l|}{7 years } \\
\hline Selective attention, $\mathrm{M}(S D)$ & $-.77(1.53)$ & $.07(.86)$ & $-.74(-1.16,-.33)$ & .001 \\
\hline Shifting attention, $\mathrm{M}(S D)$ & $-.50(1.11)$ & $.00(.92)$ & $-.49(-.82,-.17)$ & .003 \\
\hline Divided attention, $\mathrm{M}(S D)$ & $-.27(1.10)$ & $.07(.98)$ & $-.33(-.65,-.01)$ & .04 \\
\hline Processing speed, $\mathrm{M}(S D)$ & $-.55(1.18)$ & $.02(.99)$ & $-.58(-.91,-.24)$ & .001 \\
\hline Behavioral attention, $\mathrm{M}(S D)$ & $-.54(1.22)$ & $.02(.98)$ & $-.56(-.91,-.20)$ & .002 \\
\hline \multicolumn{5}{|l|}{13 years } \\
\hline Selective attention, $\mathrm{M}(S D)$ & $-.67(1.05)$ & $.04(.98)$ & $-.72(-1.02,-.41)$ & $<.001$ \\
\hline Shifting attention, $\mathrm{M}(S D)$ & $-1.01(.69)$ & $-.73(.73)$ & $-.29(-.50,-.08)$ & .006 \\
\hline Divided attention, $\mathrm{M}(S D)$ & $-.41(1.26)$ & $-.01(.96)$ & $-.40(-.75,-.05)$ & .03 \\
\hline Processing speed, M (SD) & $-.58(1.25)$ & $.00(1.00)$ & $-.58(-.94,-.23)$ & .001 \\
\hline Behavioral attention, $\mathrm{M}(S D)$ & $-.41(1.23)$ & $.02(.96)$ & $-.43(-.81,-.05)$ & .03 \\
\hline
\end{tabular}

Table 2. Fit statistics for 1 - to 5 -profile models $(n=227)$

\begin{tabular}{|c|c|c|c|c|c|c|}
\hline Number of profiles & $\mathrm{BIC}$ & Adjusted BIC & AIC & VLMR ( $p$-value) & Adjusted LMR ( $p$-value) & Entropy \\
\hline \multicolumn{7}{|l|}{7 years } \\
\hline 1 & 3466.66 & 3434.96 & 3432.41 & $\mathrm{n} / \mathrm{a}$ & $\mathrm{n} / \mathrm{a}$ & $\mathrm{n} / \mathrm{a}$ \\
\hline 2 & 3347.12 & 3296.41 & 3292.32 & .05 & .05 & .74 \\
\hline 3 & 3312.43 & 3242.71 & 3237.08 & .05 & .05 & .81 \\
\hline 4 & 3309.03 & 3220.29 & 3213.13 & .21 & .22 & .84 \\
\hline 5 & 3317.13 & 3209.37 & 3200.68 & .29 & .30 & .83 \\
\hline \multicolumn{7}{|l|}{13 years } \\
\hline 1 & 3204.30 & 3172.60 & 3170.05 & $\mathrm{n} / \mathrm{a}$ & $\mathrm{n} / \mathrm{a}$ & $\mathrm{n} / \mathrm{a}$ \\
\hline 2 & 3089.52 & 3038.81 & 3034.72 & .04 & .05 & .87 \\
\hline 3 & 3044.63 & 2974.91 & 2969.28 & .004 & .005 & .75 \\
\hline 4 & 3037.54 & 2948.80 & 2941.64 & .17 & .17 & .76 \\
\hline 5 & 3049.20 & 2941.44 & 2932.75 & .29 & .30 & .77 \\
\hline
\end{tabular}

Note. BIC = Bayesian Information Criterion; AIC = Akaike Information Criterion; VLMR = Vuong-Lo-Mendell-Rubin likelihood ratio; Adjusted LMR = adjusted Lo-Mendell-Rubin likelihood ratio test. Selected models marked in bold.

attention functioning profile at 7 years $(7 \mathrm{~b})$, VP children were more likely to transition to the corresponding profile (13b) and less likely to improve to average attentional functioning (profile 13a) compared with FT children. The extremely low to average attention functioning profile at 7 years $(7 \mathrm{c})$ only comprised VP children, of whom the majority showed partial improvement (profile 13b).

These changes were categorized into four main transition groups: (1) stable average attention pattern (i.e., $7 \mathrm{a} \rightarrow 13 \mathrm{a}$ ), (2) stable low attention pattern (i.e., $7 b \rightarrow 13 b$ or $7 \mathrm{c} \rightarrow 13 \mathrm{c}$ ),
(3) improving attention pattern (i.e., $7 \mathrm{~b} \rightarrow 13 \mathrm{a}$ or $7 \mathrm{c} \rightarrow 13 \mathrm{a} / \mathrm{b}$ ), and (4) declining attention pattern (i.e., $7 \mathrm{a} \rightarrow 13 \mathrm{~b} / \mathrm{c}$ or $7 \mathrm{~b} \rightarrow$ $13 \mathrm{c}$; Figure 3). These four transition groups were used in further analyses. Univariable analysis showed that birth status was a predictor of transition group $\left(R^{2}=.10, p<.001\right)$. Specifically, in contrast to the stable average attention pattern, VP children were more likely to have stable low attention patterns (25\% VP vs. 8\% FT children; OR, .18; 95\% CI, $.07-.50, p=.001)$, as well as improving attention patterns (23 \% VP vs. 7\% FT children; OR, .16; 95\% CI, .05-.47, 
Table 3. Comparison of attention profiles at 7 and 13 years for the total sample $(n=227)$

\begin{tabular}{|c|c|c|c|c|c|c|c|c|c|c|}
\hline & \multicolumn{10}{|c|}{ Profiles } \\
\hline & \multicolumn{5}{|c|}{7 years } & \multicolumn{5}{|c|}{13 years } \\
\hline & $7 \mathrm{a}$ & $7 b$ & $7 \mathrm{c}$ & & & $13 \mathrm{a}$ & $13 b$ & $13 \mathrm{c}$ & & \\
\hline & $n=128$ & $n=64$ & $n=35$ & $p$-value & Effect size & $n=117$ & $n=91$ & $n=19$ & $p$-value & Effect size \\
\hline Male, $n(\%)$ & $58(45)$ & $35(54)$ & $20(57)$ & .30 & .10 & $50(43)$ & $52(57)$ & $11(58)$ & .09 & .15 \\
\hline FT, $n$ ( $\%$ of FT sample) & $50(83)^{\mathrm{a}, \mathrm{b}}$ & $10(17)^{\mathrm{a}, \mathrm{c}}$ & $0^{\mathrm{b}, \mathrm{c}}$ & $<.001$ & .34 & $42(70)^{\mathrm{a}, \mathrm{b}}$ & $17(28)^{\mathrm{a}, \mathrm{c}}$ & $1(2)^{b, c}$ & .002 & .24 \\
\hline VPT, $n$ ( $\%$ of VPT sample) & $78(47)^{\mathrm{a}, \mathrm{b}}$ & $54(32)^{\mathrm{a}, \mathrm{c}}$ & $35(21)^{\mathrm{b}, \mathrm{c}}$ & $<.001$ & .34 & $75(45)^{a, b}$ & $74(44)^{\mathrm{a}, \mathrm{c}}$ & $18(11)^{\mathrm{b}, \mathrm{c}}$ & .002 & .24 \\
\hline
\end{tabular}

Note. Profiles: $7 \mathrm{a}=$ average; $7 \mathrm{~b}=$ very low-average; $7 \mathrm{c}=$ extremely low-average; $13 \mathrm{a}=$ average; $13 \mathrm{~b}=$ low average-average; $13 \mathrm{c}=$ extremely low-average. Pairwise comparison $p<.05$. Profile $1^{\mathrm{a}}$ versus $^{\mathrm{b}} ; 2^{\mathrm{a}}$ versus $^{\mathrm{c}} ; 3^{\mathrm{b}}$ versus $^{\mathrm{c}}$. Effect size: $\phi$. Small $=.10$, moderate $=.30$, large $=.50$.

(A)

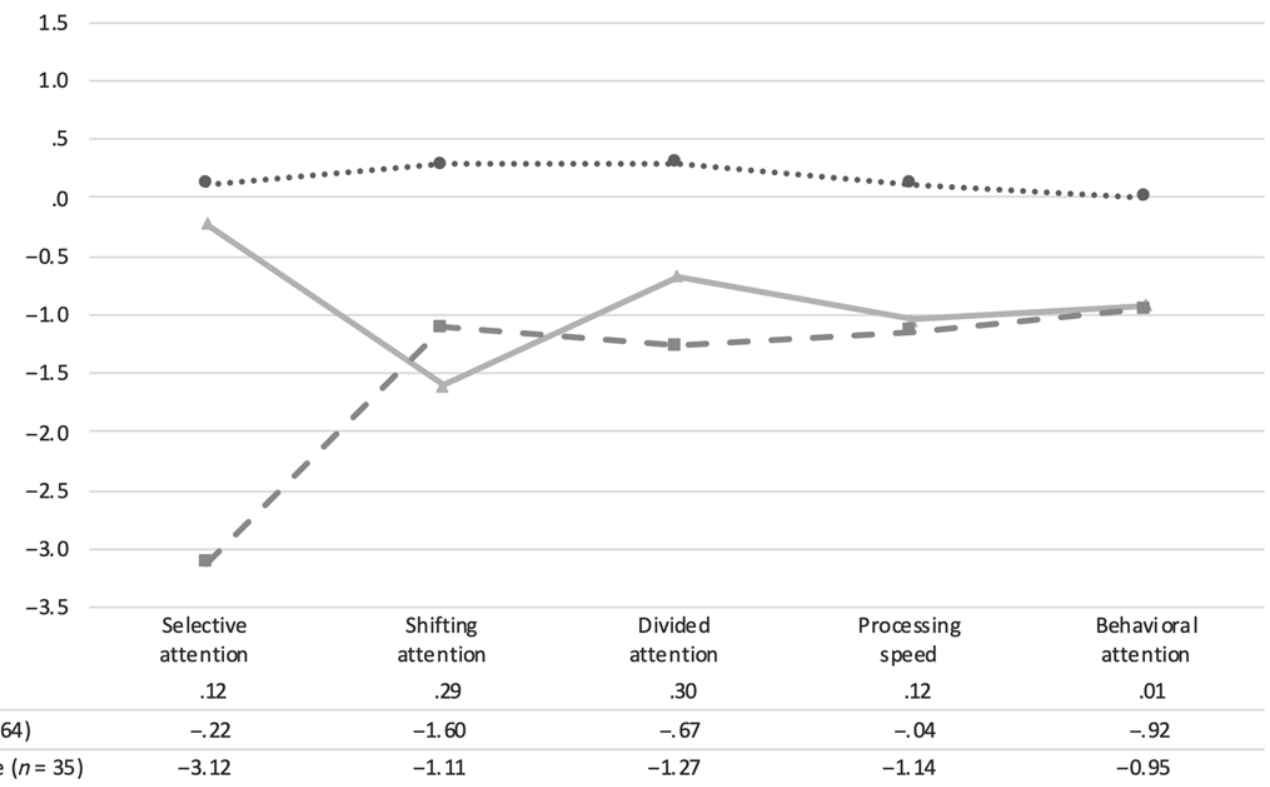

(B)

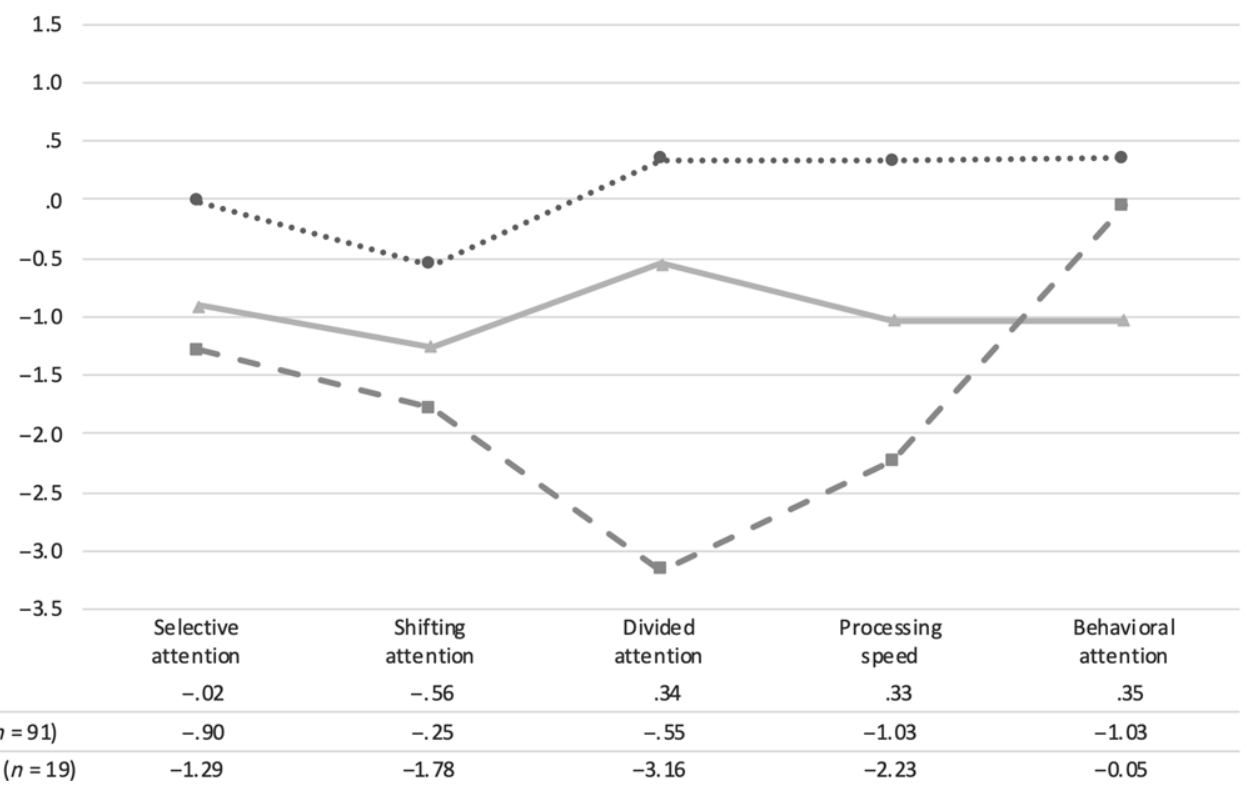

Fig. 2. Attention profiles at (A) 7 and (B) 13 years. 
Table 4. Predictors of transition groups in VP children $(n=167)$

\begin{tabular}{|c|c|c|c|c|c|c|}
\hline & $\begin{array}{l}\text { Stable poorer } v s \text {. } \\
\text { stable average }\end{array}$ & $\begin{array}{l}\text { Improving } v s \text {. } \\
\text { stable average }\end{array}$ & $\begin{array}{l}\text { Declining } v s . \\
\text { stable average }\end{array}$ & $\begin{array}{l}\text { Stable poorer } \\
v s . \text { improving }\end{array}$ & $\begin{array}{l}\text { Declining } v s . \\
\text { improving }\end{array}$ & $\begin{array}{l}\text { Stable poorer } \\
v s \text {. declining }\end{array}$ \\
\hline & OR $(95 \% \mathrm{CI})$ & OR $(95 \% \mathrm{CI})$ & OR $(95 \% \mathrm{CI})$ & OR $(95 \% \mathrm{CI})$ & OR $(95 \% \mathrm{CI})$ & OR $(95 \% \mathrm{CI})$ \\
\hline \multicolumn{7}{|l|}{ Predictors } \\
\hline Gestational age (weeks) & $.96(.71,1.31)$ & $.84(.62,1.14)$ & $1.00(.71,1.41)$ & $1.15(.85,1.54)$ & $\begin{array}{c}1.19(.85, \\
1.65)\end{array}$ & $.97(.69,1.34)$ \\
\hline Birth weight $z$-score (SDs) & $.91(.52,1.59)$ & $.84(.48,1.47)$ & $.81(.43,1.52)$ & $1.09(.61,1.94)$ & $\begin{array}{r}.96(.51 \\
1.83)\end{array}$ & $1.13(.60,2.14)$ \\
\hline $\begin{array}{l}\text { Multiple birth (twin or } \\
\text { triplet fetuses) }\end{array}$ & $.82(.33,1.99)$ & $.75(.31,1.83)$ & $1.04(.37,2.95)$ & $1.09(.44,2.70)$ & $\begin{array}{c}1.39(.49 \\
3.89)\end{array}$ & $.79(.28,2.21)$ \\
\hline $\begin{array}{l}\text { Bronchopulmonary } \\
\text { dysplasia }^{\text {a }}\end{array}$ & $.37(.12,1.14)$ & $.57(.18,1.77)$ & $.32(.09,1.15)$ & $.66(.22,1.92)$ & $\begin{array}{r}.57(.17 \\
1.88)\end{array}$ & $1.16(.35,3.84)$ \\
\hline Neonatal infection $^{\mathrm{a}}$ & $1.30(.50,3.40)$ & $2.41(.88,6.58)$ & $2.80(.87,9.00)$ & $.52(.20,1.45)$ & $\begin{array}{c}1.16(.37, \\
3.71)\end{array}$ & $.46(.15,1.42)$ \\
\hline $\begin{array}{l}\text { Cranial ultrasound major } \\
\text { brain injury }^{\mathrm{a}}\end{array}$ & $1.88(.22,16.42)$ & $1.00(.14,7.17)$ & $.37(.06,2.47)$ & $\begin{array}{r}1.88(.27 \\
13.21)\end{array}$ & $\begin{array}{c}.37(.07 \\
1.90)\end{array}$ & $\begin{array}{r}5.10(.80 \\
32.44)\end{array}$ \\
\hline $\begin{array}{l}\text { MRI brain abnormality } \\
\text { score (per unit increase) }\end{array}$ & $\begin{array}{c}1.29(1.11,1.52) \\
* *\end{array}$ & $\begin{array}{c}1.22(1.04,1.43) \\
*\end{array}$ & $\begin{array}{r}1.28(1.07 \\
1.52)^{* *}\end{array}$ & $1.06(.92,1.23)$ & $\begin{array}{c}1.05(.90, \\
1.23)\end{array}$ & $1.01(.87,1.18)$ \\
\hline Higher social risk ${ }^{\mathrm{b}}$ & $\begin{array}{c}3.51(1.40,8.78) \\
* *\end{array}$ & $\begin{array}{c}3.52(1.41,8.77) \\
* *\end{array}$ & $\begin{array}{r}7.55(2.39 \\
23.90)^{* *}\end{array}$ & $1.00(.39,2.55)$ & $\begin{array}{c}2.15(.68 \\
6.82)\end{array}$ & $.46(.15,1.48)$ \\
\hline
\end{tabular}

Note. Overall model: $R^{2}=.26, p=.002$.

${ }^{\text {a }}$ Reference $=$ no.

${ }^{\mathrm{b}}$ Reference $=$ lower; ${ }^{*} p<.05, * * p<.01$.

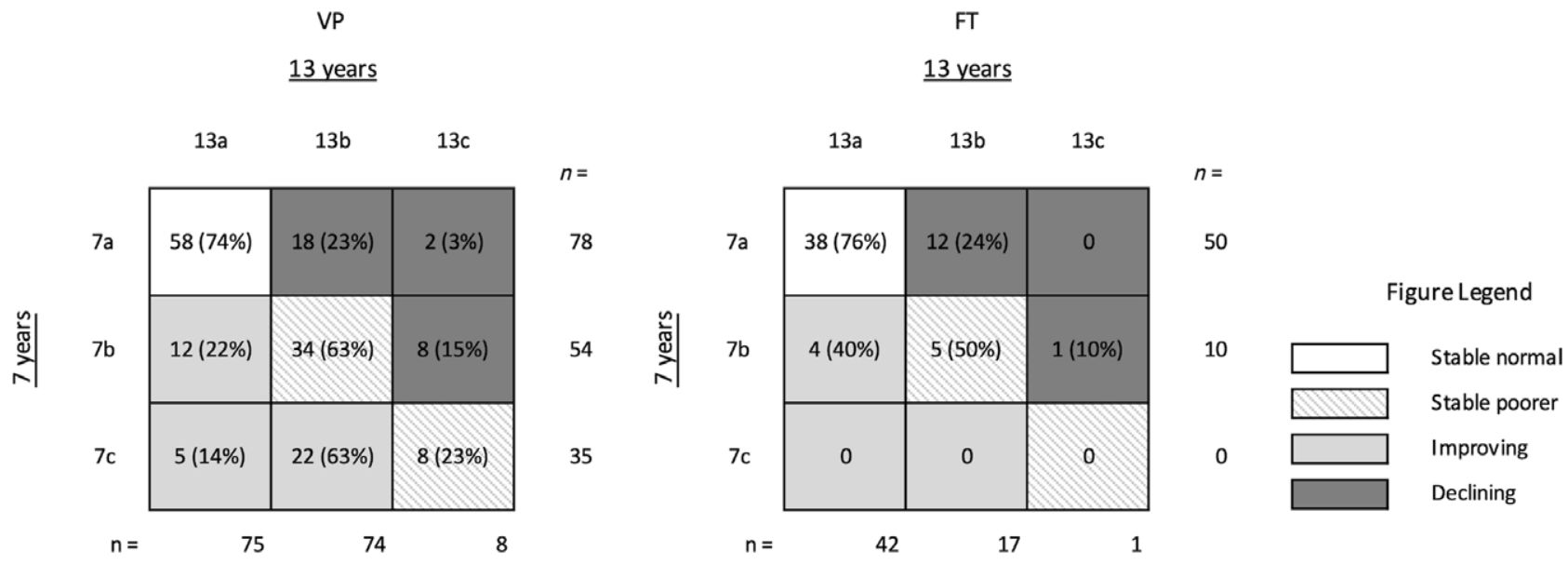

Fig. 3. VP and FT children's transitions between attention profiles from 7 to 13 years. Percentages indicate proportions of children from profile at 7 years (e.g., profile 7a) transitioning to profiles at 13 years (to 13a, 13b, or 13c).

$p=.001)$ compared with FT children. Birth status was not related to declining attention patterns (17\% VP vs. 22\% FT children; OR, .71; 95\% CI, .33-1.54, $p=.38$ ).

\section{Predictors of Transitions from 7 to 13 Years in VP Children}

For the VP group, predictors of the transition groups are shown in Table 4. Higher MRI brain abnormality scores and higher social risk at 7 years increased the odds of having a stable low or a changing (i.e., improving or declining) attention pattern compared with a stable average attention pattern. None of the risk factors examined distinguished the stable low, improving, or declining attention patterns from one another.

We also examined the associations between these predictors and attention profiles at each timepoint (Supplementary Table S2). Aligned with abovementioned results, higher MRI brain abnormality scores and higher social risk at 7 years were generally predictive of poorer attention profiles both at 7 and 13 years.

\section{DISCUSSION}

This study identified three distinct profiles of attention functioning at 7 and 13 years of age in a cohort of VP and FT 
children. At both timepoints, children born VP were more likely to exhibit a profile of poorer attentional functioning and displayed more changes between attention profiles from 7 to 13 years compared with FT children.

At both 7 and 13 years, distinct profiles of attentional functioning were identified in our sample of VP and FT children. Similar profiles were identified at both timepoints, with an average (7a and 13a) and lower to average profile (7b and 13b) emerging at both 7 and 13 years. Although profiles $7 \mathrm{c}$ and $13 \mathrm{c}$ both had poorer attentional functioning in most domains these profiles diverged over time, with specific selective attention difficulties in profile $7 \mathrm{c}$, and marked difficulties in shifting attention, divided attention, and processing speed in profile $13 \mathrm{c}$. At 7 years, children in profile $7 \mathrm{c}$ were differentiated from children in profile $7 \mathrm{~b}$ by displaying substantial difficulties in selective attention, while otherwise performing similarly across the other attention domains (Figure 3). Notably, profile 7c consisted only of VP children, indicating delayed selective attention development in a subset of VP children specifically. Given that the 7-year selective attention task in our study required children to perform a visual search, poor performance could reflect an immature, inefficient search pattern (Datin-Dorrière et al., 2020; Geldof et al., 2013) or delayed development of planning (an executive functioning component) in some VP children (Loe, Heller, \& Chatav, 2019). At 13 years, none of the profiles were characterized specifically by selective attention difficulties. While profile $13 \mathrm{c}$ showed somewhat lower selective attention performance, this profile was distinguished more clearly by difficulties in shifting attention, divided attention, and processing speed. Thus, at 7 years performance on selective attention differentiated children, but at 13 years performance on this attention domain was no longer the most distinctive. This suggests VP children's relative strengths and weaknesses in attentional functioning are not fixed throughout childhood, which may explain inconsistent findings across past studies, and has implications for how VP children's attentional functioning is monitored and managed.

At both timepoints, the majority of FT children demonstrated a profile of average attentional functioning, in contrast to less than half of the VP children. VP children were 2-3 times more likely to have a profile of generalized lower attentional functioning (profiles $7 \mathrm{~b}$ and $13 \mathrm{~b}$ ) or a profile marked by specific attention difficulties (profiles 7c and 13c) compared with FT children. These findings are largely in accordance with a previous study reporting that 6 -year-old children born moderate-to-late preterm also showed several distinct attention profiles, and were more likely to have poorer profiles of attentional functioning compared with FT born children (Bogičević et al., 2020).

From 7 to 13 years, VP children showed more individual variability in transition patterns between attention profiles compared with FT children. The majority of FT children had stable average attentional functioning in contrast to only one-third of VP children (63\% vs. $35 \%$ ). VP children were roughly three times more likely to have a stable low attention pattern compared with FT children. Optimistically, VP children were also three times more likely to have an improving attention pattern, and they were not at increased risk for a declining attention pattern. This can partially be explained by the finding that at 7 years, VP children were at increased risk of having a poorer attention profile and hence it is less likely for their relative performance to decline. By 13 years of age, nearly half of all VP children were classified within a profile of optimal attentional functioning, of which some children showed an initial delay with subsequent catch-up. However, still $42 \%$ of VP children exhibited stable low or declining attention difficulties, indicating the long-term effects of VP birth on attentional functioning. Studies that have examined variability in VP children's longitudinal outcomes in other areas of cognitive functioning generally report similar proportions of VP children with optimal (46-65\%) versus adverse outcomes (35-54\%; Luu, Vohr, Allan, Schneider, \& Ment, 2011), including language functioning assessed in the current cohort from 2 to 13 years of age (Nguyen et al., 2018).

To understand the mechanisms underlying individual variability in outcomes and distinguish optimally developing VP children from those who are more vulnerable, we considered potential biological and social risk factors as predictors of transition patterns. We found that VP children with improving, stable low, or declining attention patterns had more severe brain abnormalities at term-equivalent age and were more likely to come from a less advantaged family environment at 7 years of age than children who had stable average attentional functioning over time. VP children with improving attentional functioning had somewhat less severe neonatal brain abnormalities and lower social risk than VP children with adverse attention patterns (stable low and declining attention). However, these factors did not significantly distinguish VP children with improving patterns from those with stable low or declining attention difficulties. In other words, early brain pathology and a less advantaged family environment were markers for poorer attentional functioning during some point throughout late childhood, rather than predicting variability in attention patterns over time. A recent study reported a high degree of heterogeneity in preterm born children's brain development on neonatal MRI, with a higher rate of deviation from typical development predicting poorer cognitive outcomes at toddler age (Dimitrova et al., 2020). The link between structural and functional brain development and heterogeneity in VP children's attentional functioning should also be addressed in future studies. At least some VP children displayed (partial) catch-up in attentional functioning over time, and this improvement could not be explained by the predictors examined in this study. Previous research found other child and family factors, such as early childhood skills and temperament, parental well-being, and parenting practices, as protective factors for preterm born children's development (Lean et al., 2020; Poehlmann-Tynan et al., 2015). Indeed, de Jong et al. (2018) demonstrated that maternal stimulation of attention behaviors (i.e., more maternal redirecting of the child's attention) was associated with poorer attentional 
functioning in moderate-to-late preterm born toddlers', which in turn was related to poorer general cognitive functioning. Future research should investigate the mechanisms underlying variability in the development of attention patterns to further identify VP children at highest risk of adverse attentional functioning, as well as target interventions at potentially modifiable protective factors.

The current study has several implications. Our results suggest that assessments of attentional functioning in VP children should ideally include multiple attention domains, as VP children may show profiles with specific, clinically important, attention difficulties. Moreover, as a large number of VP children had a profile of generalized subaverage attentional functioning, the pattern of functioning across these attention domains needs to be evaluated simultaneously. Poorer functioning across multiple attention domains may have a great impact on functioning in other areas, such as IQ and academic achievement (Rose, Feldman, \& Jankowski, 2011; Rose et al., 2011). In addition, transition patterns for VP children specifically showed that having a profile of subaverage attention at 7 years is a marker for poorer attention outcomes at 13 years. This finding emphasizes the need for strategies to improve attentional functioning before 7 years of age.

A strength of this study is that we addressed VP children's heterogeneity in comparison with a FT control group, and in relation to biological and social risk factors. Other strengths include a longitudinal design with good retention rates, and a multi-informant, multifaceted measurement of attention repeated consistently over time, allowing for a comprehensive assessment of VP children's patterns of attentional functioning. To capture the different developmental progressions of attentional functioning, it was important to use ageappropriate measures at each timepoint. While the underlying concepts of the measures at 7 years correspond to those at 13 years, some differences in how these concepts were measured at the two timepoints may have partially affected structural changes in profiles over time. Although we examined multiple attention domains, sustained attention was not included in this study because our measure at 13 years was not age-appropriate. Considering that sustained attention has previously found to be affected in VP children (e.g., Lean et al., 2017), further studies should include ageappropriate measures in assessment of attentional functioning. Another limitation was that in the 3-profile solution at 13 years, entropy was moderate rather than good, meaning that assignment to profiles may have been somewhat less precise for the sample at 13 years than at 7 years.

Our study demonstrated individual variability in the profiles of attentional functioning in VP children as well as heterogenous development of attention throughout late childhood. VP children have a two- to threefold risk of a poorer profile of attentional functioning, with lower generalized attention or specific attention difficulties, compared with FT peers. VP children showed more change between attention profiles over time, and the pattern of attention difficulties in VP children may evolve with age. While almost half of VP children improved in attentional functioning by 13 years of age, the remaining half of VP children, with the highest rates of early brain pathology and a less advantaged social environment, experienced stable low or declining differentiated attention difficulties. These findings highlight the need for long-term individualized strategies to improve attentional functioning.

\section{SUPPLEMENTARY MATERIAL}

To view supplementary material for this article, please visit https://doi.org/10.1017/S1355617720001411

\section{ACKNOWLEDGEMENTS}

We want to thank all the families who participated in this study and the entire VIBeS research team.

\section{FINANCIAL SUPPORT}

This work was supported by Australia's National Health \& Medical Research Council (Project grants 237117, 491209, 1066555; Research Excellence in Newborn Medicine to A.C.B., J.L.Y.C., L.W.D., P.J.A., 1153176; Senior Research Fellowship to P.J.A., 1081288; Leadership Fellowship to P.J.A., 1176077); the Prins Bernhard Cultuurfonds to L.B., 40026915; Stichting Jo Kolk Studiefonds to L.B.; the Medical Research Future Fund Career Development Fellowship to J.L.Y.C., 1141354; Utrecht University; Murdoch Children's Research Institute; Monash University; and the Victorian Government's Operational Infrastructure Support Program.

\section{CONFLICT OF INTEREST}

The authors have no conflicts of interest to declare.

\section{REFERENCES}

Aarnoudse-Moens, C.S., Weisglas-Kuperus, N., van Goudoever, J.B., \& Oosterlaan, J. (2009). Meta-Analysis of neurobehavioral outcomes in very preterm and/or very low birth weight children. Pediatrics, 124(2), 717-728. doi: 10.1542/peds.2008-2816

Anderson, P.J., Anderson, V., Northam, E., \& Taylor, H.G. (2000). Standardization of the contingency naming test (CNT) for schoolaged children: a measure of reactive flexibility. Clinical Neuropsychological Assessment, 1, 247-273.

Anderson, P.J., De Luca, C.R., Hutchinson, E., Spencer-Smith, M.M., Roberts, G., Doyle, L.W., \& Victorian Infant Collaborative Study Group (2011). Attention problems in a representative sample of extremely preterm/extremely low birth weight children. Developmental Neuropsychology, 36(1), 57-73. doi: 10.1080/ 87565641.2011.540538

Anderson, P.J. \& Doyle, L.W. (2003). Neurobehavioral outcomes of school-age children born extremely low birth weight or very preterm in the 1990s. Journal of the American Medical Association, 289, 3264-3272. doi: 10.1001/jama.289.24.3264 
Bayless, S. \& Stevenson, J. (2007). Executive functions in schoolage children born very prematurely. Early Human Development, 83(4), 247-254. doi: 10.1016/j.earlhumdev.2006.05.021

Berlin, K.S., Williams, N.A., \& Parra, G.R. (2014). An introduction to latent variable mixture modeling (part 1): overview and crosssectional latent class and latent profile analyses. Journal of Pediatric Psychology, 39(2), 174-187. doi: 10.1093/jpepsy/ jst084

Bogicevic, L., Verhoeven, M., \& van Baar, A.L. (2020). Distinct profiles of attention in children born moderate-to-late preterm at 6 years. Journal of Pediatric Psychology, 45(6), 685-694. doi: 10.1093/jpepsy/jsaa038

Breeman, L.D., Jaekel, J., Baumann, N., Bartmann, P., \& Wolke, D. (2016). Attention problems in very preterm children from childhood to adulthood: the Bavarian Longitudinal Study. Journal of Child Psychology and Psychiatry, 57(2), 132-140. doi: 10.1111/ jcpp. 12456

Burnett, A.C., Youssef, G., Anderson, P.J., Duff, J., Doyle, L.W., \& Cheong, J.L.Y. (2019). Exploring the "preterm behavioral phenotype" in children born extremely preterm. Journal of Developmental \& Behavioral Pediatrics, 40(3), 200-207. doi: 10.1097/DBP.0000000000000646

Datin-Dorriere, V., Borst, G., Guillois, B., Cachia, A., \& Poirel, N. (2020). The forest, the trees, and the leaves in preterm children: the impact of prematurity on a visual search task containing three-level hierarchical stimuli. European Child \& Adolescent Psychiatry. doi: 10.1007/s00787-020-01510-x

de Jong, M., Verhoeven, M., Hooge, I.T.C., Maingay-Visser, A., Spanjerberg, L., \& van Baar, A.L. (2018). Cognitive functioning in toddlerhood: the role of gestational age, attention capacities, and maternal stimulation. Developmental Psychology, 54(4), 648-662. doi: 10.1037/dev0000446

Dimitrova, R., Pietsch, M., Christiaens, D., Ciarrusta, J., Wolfers, T., Batalle, D., ... O'Muircheartaigh, J. (2020). Heterogeneity in brain microstructural development following preterm birth. Cerebral Cortex, bhaa069, 1-11. doi: 10.1093/cercor/bhaa069

Eryigit-Madzwamuse, S., \& Wolke, D. (2015). Attention problems in relation to gestational age at birth and smallness for gestational age. Early Human Development, 91(2), 131-138. doi: 10.1016/j. earlhumdev.2015.01.004

Espy, K.A. (1997). The shape school: assessing executive function in preschool children. Developmental Neuropsychology, 13(4), 495-499. doi: 10.1080/87565649709540690

Geldof, C.J., de Kieviet, J.F., Dik, M., Kok, J.H., van WassenaerLeemhuis, A.G., \& Oosterlaan, J. (2013). Visual search and attention in five-year-old very preterm/very low birth weight children. Early Human Development, 89(12), 983-988. doi: 10.1016/j. earlhumdev.2013.08.021

Goodman, R. (1997). The strengths and difficulties questionnaire: a research note. Journal of Child Psychology and Psychiatry, 38, 581-586. doi: 10.1111/j.1469-7610.1997.tb01545.x

Kidokoro, H., Neil, J.J., \& Inder, T.E. (2013). New MR imaging assessment tool to define brain abnormalities in very preterm infants at term. American Journal of Neuroradiology, 34(11), 2208-2214. doi: 10.3174/ajnr.A3521

Krasner, A.J., Turner, J.B., Feldman, J.F., Silberman, A.E., Fisher, P.W., Workman, C.C., ... Whitaker, A.H. (2018). ADHD symptoms in a non-referred low birthweight/preterm cohort: longitudinal profiles, outcomes, and associated features. Journal of Attention Disorders, 22(9), 827-838. doi: $10.1177 / 1087054715617532$
Lean, R.E., Lessov-Shlaggar, C.N., Gerstein, E.D., Smyser, T.A., Paul, R.A., Smyser, C.D., \& Rogers, C.E. (2020). Maternal and family factors differentiate profiles of psychiatric impairments in very preterm children at age 5-years. Journal of Child Psychology and Psychiatry, 61(2), 157-166. doi: 10.1111/jcpp. 13116

Lean, R.E., Melzer, T.R., Bora, S., Watts, R., \& Woodward, L.J. (2017). Attention and regional gray matter development in very preterm children at age 12 years. Journal of the International Society or Neuropsychology, 23(7), 539-550. doi: 10.1017/ S1355617717000388

Loe, I.M., Heller, N.A., \& Chatav, M. (2019). Behavior problems and executive function impairments in preterm compared to full term preschoolers. Early Human Development, 130, 87-95. doi: 10.1016/j.earlhumdev.2019.01.014

Luu, T.M., Vohr, B.R., Allan, W., Schneider, K.C., \& Ment, L.R. (2011). Evidence for catch-up in cognition and receptive vocabulary among adolescents born very preterm. Pediatrics, 128(2), 313-322. doi: 10.1542/peds.2010-2655

Manly, T., Anderson, V., Nimmo-Smith, I., Turner, A., Watson, P., \& Robertson, I.H. (2001). The differential assessment of children's attention: the test of everyday attention for children (TEA-Ch), normative sample and ADHD performance. Journal of Child Psychology and Psychiatry, 42, 1065-1081. doi: 10.1111/ 1469-7610.00806

Manly, T., Robertson, I.H., Anderson, V., \& Nimmo-Smith, I. (1999). TEA-Ch: The test of everyday attention for children. Bury St. Edmunds, UK: Thames Valley Test Company.

McGrath, M.M., Sullivan, M., Devin, J., Fontes-Murphy, M., Barcelos, S., DePalma, J.L., \& Faraone, S. (2005). Early precursors of low attention and hyperactivity in a preterm sample at age four. Issues in Comprehensive Pediatric Nursing, 28(1), 1-15. doi: 10.1080/01460860590913945

Mulder, H., Pitchford, N.J., Hagger, M.S., \& Marlow, N. (2009). Development of executive function and attention in preterm children: a systematic review. Developmental Neuropsychology, 34(4), 393-421. doi: 10.1080/87565640902964524

Mulder, H., Pitchford, N.J., \& Marlow, N. (2011). Processing speed mediates executive function difficulties in very preterm children in middle childhood. Journal of the International Neuropsychological Society, 17(3), 445-454. doi: 10.1017/ s1355617711000373

Murray, A.L., Scratch, S.E., Thompson, D.K., Inder, T.E., Doyle, L.W., Anderson, J.F.I., \& Anderson, P.J. (2014). Neonatal brain pathology predicts adverse attention and processing speed outcomes in very preterm and/or very low birth weight children. Neuropsychology, 28(4), 552-562. doi: 10.1037/neu0000071

Muthén, L.K. and Muthén, B.O. (1998-2012). Mplus User's Guide (7th ed.). Los Angeles, CA: Muthén \& Muthén.

Nguyen, T.N., Spencer-Smith, M., Haebich, K.M., Burnett, A., Scratch, S.E., Cheong, J.L.Y., _. Anderson, P.J. (2018). Language trajectories of children born very preterm and full term from early to late childhood. Journal of Pediatrics, 202, 86-91. doi: 10.1016/j.jpeds.2018.06.036

Poehlmann-Tynan, J., Gerstein, E.D., Burnson, C., Weymouth, L., Bolt, D.M., Maleck, S., \& Schwichtenberg, A.J. (2015). Risk and resilience in preterm children at age 6 . Developmental Psychopathology, 27(3), 843-858. doi: 10. 1017/S095457941400087X

Roberts, G., Howard, K., Spittle, A.J., Brown, N.C., Anderson, P.J., $\&$ Doyle, L.W. (2008). Rates of early intervention services in very 
preterm children with developmental disabilities at age 2 years. Journal of Paediatrics and Child Health, 44(5), 276-280. doi: 10.1111/j.1440-1754.2007.01251.x

Rose, S.A., Feldman, J.F., \& Jankowski, J.J. (2011). Modeling a cascade of effects: the role of speed and executive functioning in preterm/full-term differences in academic achievement.
Developmental Science, 14(5), 1161-1175. doi: 10.1111/j. 1467-7687.2011.01068.x

Rose, S.A., Feldman, J.F., Jankowski, J.J., \& Van Rossem, R. (2011). Basic information processing abilities at 11 years account for deficits in IQ associated with preterm birth. Intelligence, 39(4), 198-209. doi: 10.1016/j.intell.2011.03.003 